\title{
Simple A Posteriori Error Estimators for the $h$-Version of the Boundary Element Method
}

\author{
* Samuel Ferraz-Leite ${ }^{1}$ and Dirk Praetorius ${ }^{2}$ \\ ${ }^{1}$ Vienna University of Technology \\ Institute for Analysis and Scientific Computing, \\ Wiedner Hauptstraße 8-10, 1040 Wien, Austria \\ samuel.ferraz-leite@tuwien.ac.at \\ http://www.asc.tuwien.ac.at/ samuel \\ 2 Vienna University of Technology \\ Institute for Analysis and Scientific Computing, \\ Wiedner Hauptstraße 8-10, 1040 Wien, Austria \\ dirk.praetorius@tuwien.ac.at \\ http://www.math.tuwien.ac.at/ dirk
}

Key Words: BEM, a posteriori error estimation, adaptive mesh refinement, Symm's integral equation.

Key Words. BEM, a posterioni error estimation, adaptive mesh refinement, Symm's integral equation.

\begin{abstract}
The $h$ - $h / 2$-strategy is one very basic and well-known technique for the a posteriori error estimation for Galerkin discretizations of energy minimization problems. Let $\phi$ denote the exact solution. One then considers
\end{abstract}

$$
\eta_{H}:=\left\|\phi_{h}-\phi_{h / 2}\right\|
$$

to estimate the error $\left\|\phi-\phi_{h}\right\|$, where $\phi_{h}$ is a Galerkin solution with respect to a mesh $\mathcal{T}_{h}$ and $\phi_{h / 2}$ is a Galerkin solution for a mesh $\mathcal{T}_{h / 2}$ obtained from uniform refinement of $\mathcal{T}_{h}$. We stress that $\eta_{H}$ is always efficient - even with known efficiency constant $C_{\text {eff }}=1$, i.e.

$$
\eta_{H} \leq\left\|\phi-\phi_{h}\right\|
$$

Reliability of $\eta_{H}$ follows immediately from the assumption $\left\|\phi-\phi_{h / 2}\right\| \leq \sigma\left\|\phi-\phi_{h}\right\|$ with some saturation constant $\sigma \in(0,1)$. Under this assumption, there holds

$$
\left\|\phi-\phi_{h}\right\| \leq \frac{1}{\sqrt{1-\sigma^{2}}} \eta_{H}
$$

However, for boundary element methods, the energy norm $\|\cdot\|$ is non-local and thus the error estimator $\eta_{H}$ does not provide information for a local mesh-refinement. Recent localization techniques from [1] for $\widetilde{H}^{-\alpha}$-norms and [3] for $\widetilde{H}^{\alpha}$-norms allow to replace the energy norm in this case by $h$-weighted $L^{2}$-norms resp. $H^{1}$-norms, where $h$ denotes the local mesh-size. In particular, this very basic error estimation strategy is also applicable to steer an $h$-adaptive mesh-refinement. For instance, for Symm's integral equation, the $L^{2}$-norm based estimator

$$
\mu_{H}:=\left\|h^{1 / 2}\left(\phi_{h}-\phi_{h / 2}\right)\right\|_{L^{2}(\Gamma)}
$$

is equivalent to $\eta_{H}$. We thus may use $\mu_{H}$ to steer the mesh and $\eta_{H}$ to estimate the error. 
Further simplifications of the proposed error estimators $\eta_{H}$ and $\mu_{H}$ consist of replacing $\phi_{h}$ by some appropriate projection $\Pi_{h} \phi_{h / 2}$, for instance, by use of the $L^{2}$-projection onto the discrete space corresponding to $\mathcal{T}_{h}$.

Moreover, the error estimator $\eta_{H}$ is proven to be equivalent to the averaging estimator in [4] and the two level estimator from [5].

Numerical experiments in 2D and 3D for first-kind integral equations with weakly-singular integral operator conclude the talk.

\section{REFERENCES}

[1] C. Carstensen, D. Praetorius. Averaging techniques for the effective numerical solution of Symm's integral equation of the first kind, SIAM J.Sci.Comp. 27 (2006), 1226-1260.

[2] S. Ferraz-Leite, D. Praetorius. Simple A Posteriori Error Estimators for the h-Version of the Boundary Element Method, ASC Report 01/2007, Institute for Analysis and Scientific Computing, Vienna University of Technology, Wien, 2007

[3] S. Funken, S. Ferraz-Leite, D. Praetorius. Averaging on large patches for integral equations in $3 D$, work in progress (2007)

[4] C. Erath, S. Funken, S. Ferraz-Leite, D. Praetorius. Energy norm based a posteriori error estimation for boundary element methods in two dimensions, ASC Report 07/2007, Institute for Analysis and Scientific Computing, Vienna University of Technology, Wien, 2007

[5] P. Mund, E. P. Stephan, J. Weisse. Two-level methods for the single layer potential in $\mathbb{R}^{3}$, Computing 60 (1998), 243-266. 